Research Article

\title{
Diet Quality Indices and Their Correlation with Glycemic Status and Lipid Profile in Patients with Type 2 Diabetes
}

\author{
Roxaneh Sadat Ziaee, ${ }^{1}$ Parisa Keshani $\mathbb{D}^{2},{ }^{2}$ Moosa Salehi, ${ }^{1}$ and Haleh Ghaem ${ }^{3}$ \\ ${ }^{1}$ School of Nutrition and Food Sciences, Shiraz University of Medical Sciences, Shiraz, Iran \\ ${ }^{2}$ Shiraz HIV/AIDS Research Center, Institute of Health, Shiraz University of Medical Sciences, Shiraz, Iran \\ ${ }^{3}$ Department of Epidemiology, School of Health, Shiraz University of Medical Sciences, Shiraz, Iran \\ Correspondence should be addressed to Parisa Keshani; parisa.keshani@gmail.com
}

Received 13 March 2020; Revised 18 August 2020; Accepted 1 March 2021; Published 8 March 2021

Academic Editor: Diego A. S. Silva

Copyright ( $\odot 2021$ Roxaneh Sadat Ziaee et al. This is an open access article distributed under the Creative Commons Attribution License, which permits unrestricted use, distribution, and reproduction in any medium, provided the original work is properly cited.

\begin{abstract}
Background. The study aimed to determine the correlation between different diet quality indices and glycemic status and lipid profile in patients with diabetes. Methods. This study was carried out on 235 patients with type 2 diabetes referred to Martyr Motahari Diabetes Clinic of Shiraz city so as to investigate the relationship between glycemic status and lipid profile and the diet quality using Healthy Eating Index (HEI-2010), phytochemical index (PI), and Diet Quality Index-International (DQI_I). Results. A positive correlation was indicated between the serum levels of LDL-C and HEI-2010 scores $(p=0.026)$. Furthermore, there was a positive correlation between the patients' age and scores of PI $(p=0.006)$ and between PI and DQI_I $(p<0.001)$. There was no significant relationship between the scores for all three indicators and biochemical parameters. Conclusion. The results of this study indicated that there was a significant correlation between the serum LDL-C levels and the HEI-2010 scores. Moreover, the age of the patients had a direct correlation with the PI scores.
\end{abstract}

\section{Introduction}

Type 2 diabetes is a complex and chronic disease defined by a heterogeneous set of metabolic disorders including deficit in carbohydrate metabolism, protein, and lipid [1]. Nowadays, type 2 diabetes is one of the most challenging health problems around the world because of its high incidence, mortality caused by the disease, and the costs related to its treatment; there is a need for timely attention and monitoring in order to prevent the disease development and its better control [2]. Nutritional education and increase in nutritional awareness of patients can stop the developing process of type 2 diabetes or slow it down. Nowadays, various valid diet quality indices including Healthy Diet Indicator (HDI), Diet Quality Index (DQI), Alternate HEI (AHEI), Healthy Eating Index (HEI), DQI-International (DQI-I), and Mediterranean Diet Score have been introduced and developed [3]. Various studies have been conducted, indicating the correlation between diet quality and chronic diseases, especially cancer and cardiovascular diseases. Some researchers also have shown that there is a correlation between the quality indices and obesity and diabetes [4-7]. However, few studies are conducted on the correlation between diet quality indices and glycemic control and lipid profile; also, up to now, no studies have been carried out to evaluate the correlation between various indices. Diet quality indices might be used as a rapid evaluator of healthy nutritional diets after education of diet. With regard to the role of diet and/or medical nutrition treatment in chronic diseases, the approach of diet quality which includes adequate indices in diet is suggested as a powerful tool because of the complexity of diets. The HEI-2010 and the DQI-I are most common and used indices to assess diet in patients with noncommunicable diseases based on different items including macronutrients and micronutrients. Plantextracted substances such as phytochemicals have been suggested to have antidiabetic activity [8]. Also, numerous studies have reported that diabetes mellitus causes more free 
radicals and decreases antioxidant parameters and capacity. Therefore, in some studies using phytochemicals is recommended to progress the insulin sensitivity [9]. Phytochemical index (PI) is an index to assess these compounds in foods [10]. In this way, the aim of the present study was to evaluate the correlation of three diet quality indices with glycemic status and lipid profile in patients with type 2 diabetes.

\section{Materials and Methods}

2.1. Participants. Using all three indices, the higher sample size was calculated by HEI considering $\mathrm{SD}=12.5, \alpha=0.05$, and $d=1.6$ [11]. The subjects included 235 patients with type 2 diabetes and on oral-antidiabetic medications who were selected by convenient sampling method, from patients referred to Shiraz Motahari Diabetes Clinic. Inclusion criteria were known case of type 2 diabetes for one to 10 years, and age 30-65 years old. Patients who had diabetes related complications (neuropathy, nephropathy, or atherosclerosis), organ dysfunctions, recent surgery, and pregnancy and lactation or using immunosuppressive and immunoregulatory drug or insulin were excluded from the study. Firstly, the aims and stages of study were completely described for them and after justifying the participants, written consent forms were completed. This study was approved by ethical commission in Shiraz University of Medical Sciences no. IR.SUMS.REC.1394.S637.

\subsection{Demographic, Anthropometric, and Clinical Parameters.} Demographic information including gender (male, female), education (illiterate, primary school, secondary school, high school and diploma, and university), employment (unemployed and housekeeper, employed, and retired), and marital status (single, married, widow, and divorced), and their physical activity was asked of the participants. At least 150 to 300 minutes a week of moderate-intensity physical activity, or 75 to 150 minutes in a week of vigorous-intensity physical activity, is considered as physically active in this study [12].

Height was measured to the nearest centimeter, while the subjects were barefoot, and body weight was measured to the nearest $0.1 \mathrm{~kg}$ using a digital scale, while the subjects wore light clothing. Then, body mass index (BMI) was calculated by determining the ratio between weight and height squared $(\mathrm{kg} / \mathrm{m} 2)$. Blood pressure was recorded using a mercury sphygmomanometer twice in a sitting position after the subjects were in a relaxed state for at least 15 minutes. Again, after 5 minutes it was measured from the right arm and the average of two measurements were recorded as the final rate of blood pressure.

Venous blood samples $(10 \mathrm{cc})$ were taken after 12 hours overnight fasting. The blood samples taken were immediately centrifuged and the serum was separated and maintained in -70 centigrade degrees up to the trial time. At the end of the study, the rates of glucose concentrations, insulin, glycosylated hemoglobin, triglyceride, total cholesterol, and HDL and LDL cholesterol were measured by commercial kits. Insulin and hemoglobin $A_{1} c$ were measured by Human Insulin ELISA kit (PARS AZMUN) and high-performance liquid chromatography kits (PARS AZMUN), respectively. Insulin resistance was calculated using the following formula:

insulin resistance $=($ fasting insulin $(\mathrm{mIU} / \mathrm{L}) \times$ fasting glucose $(\mathrm{mmol} / \mathrm{L})) / 22.5$.

2.3. Dietary Assessment. Data about nutrition received by the patients were collected using the 24-hour dietary recall related to three days of the week (first day of week, midweek, and end of week). A 24-hour dietary recall is an organized interview, planned to collect information about all foods and beverages, ingredients, and the amount consumed by the respondent in the past 24 hours $[13,14]$.

On the visiting day, the first 24-hour recall was asked from the patients; also, the second and third reminders were asked from them by telephone and completed. The energy and macronutrients uptake were measured using the NUTRITIONIST-4 software and revised according to Iranian diet. With regard to not completing the table of Iranian compounds for the number of nutritional items and micronutrients, USDA table of nutritional compounds was also used in order to further analyze the nutritional items for receiving energy and nutrition. Common daily nutrition uptake of the patients was evaluated according to the household modules and then the daily received amounts were converted to gram. For mixed foods, the nutrition was also calculated according to the sum of the nutrition of the ingredients of that food. Finally, the data were used for measurement of the quality of the diet for each individual. Then, it was calculated according to HEI-2010, DQI-I, and PI indices, using the results of three 24-hour nutrition recall questionnaires.

\subsection{Application of the HEI-2010, DQI-I, and PI to the Datasets.} The HEI-2010 has 12 food components, 9 adequacy components with higher scores indicating higher consumption, and 3 moderation components with higher scores indicating lower consumption. The adequacy components include total fruit (5 points); whole fruit (5 points); total vegetables (5 points); greens and beans (5 points); whole grains (10 points); dairy (10 points); total protein foods (5 points); seafood and plant proteins (5 points); and fatty acids (10 points). The components that should be served in moderation include refined grains (10 points); sodium (10 points); and empty calories (solid fats, alcoholic beverages, and added sugars) (20 points). The mentioned scores were adjusted for energy (per 1,000 kcal or as a percentage of energy), except for the fatty acids ratio. The total HEI-2010 score which has a maximum value of 100 is a sum of all components, with higher total scores reflecting better diet quality [15].

The DQI-I focuses on 4 major aspects: variety (0-20 points), adequacy (0-40 points), moderation ( $0-30$ points), and overall balance (0-10 points). The scores for all 4 categories were totaled, from 0 to 100 ( 0 being the worst and 100 the best possible scores) [16]. 
The dietary PI was calculated based on the method developed by McCarty [17] PI = [daily energy derived from phytochemical-rich foods $\mathrm{kJ}$ (kcal)/total daily energy intake $\mathrm{kJ}(\mathrm{kcal})]^{*} 100$. Foods included in the phytochemical-rich category were fruits and vegetables, legumes, whole grains, seeds, nuts, natural fruit and vegetable juices, olive oil, and soy products.

2.5. Statistical Analysis. Due to the normal distribution of data, the mean and standard deviation was used to describe quantitative data and the frequency and percentage for qualitative variables. We used Kolmogrov-Smirnov and Shapiro-wilk tests to check the normality and Pearson tests to evaluate the correlation between variables as needed. Considering the significance level of 0.05 , SPSS software version 20 was used to analyze the data and NUTRITIONIST-4 software was used to evaluate the nutritional status and extraction of micronutrients and macronutrients.

\section{Results}

From the 235 patients, 148 (63\%) were men and $87(27 \%)$ were women. The mean age of the patients was $55.11 \pm 7.89$ years. The average values of height, weight, body mass index, blood pressure, and metabolic indices of patients are shown in Table 1 . The results showed that there was a significant positive correlation of PI with age and HDL; on the other hand, there was a significant reverse correlation between BMI of patients and PI. There was no significant correlation between the variables evaluated with DQI-I index. However, total cholesterol levels and LDL-C levels of patients had a direct and significant correlation with HEI-2010 score (Table 2). The mean scores for DQI-I, HEI-2010, and PI in this study were $58.82 \pm 7.34$ (Table 3 ), $55.83 \pm 10.61$ (Table 4), and $36.79 \pm 15.99$.

\section{Discussion}

The evaluation of diet based on diet quality indices offers us a more comprehensive approach about the correlation of nutrition receiving and health, compared to evaluation of receiving nutrition only. Inappropriate diet quality and absence of physical activity have led to increase in the obesity incidence and thereupon type 2 diabetes in developed and developing countries [18]. Also, it has been observed that high-quality diet is related to a decrease in the mortality risk caused by cardiovascular diseases and a decrease in the risk of type 2 diabetes [19]. Type 2 diabetes is one of the most challenging health problems around the world which needs timely attention and follow-up in order to prevent the development of the disease and its better control. The evaluation of the diet quality and recognition of appropriate index related to better glycemic control and better status of lipid profile can be used for presentation of applied and effective nutritional recommendations in order to remove the existing nutritional problems. In Iran, a limited number of studies have been conducted on diet quality indices, especially in diabetic patients, and the three indices of diet quality
TABle 1: Demographic and clinical characteristics of participants $(n=235)$.

\begin{tabular}{lc}
\hline Variables & Mean \pm SD \\
\hline Age & $55.11 \pm 7.89$ \\
BMI & $27.02 \pm 3.66$ \\
BP-systolic & $127.38 \pm 15.30$ \\
BP-diastolic & $81.52 \pm 7.19$ \\
TG (mg/dl) & $193.96 \pm 79.54$ \\
Insulin $(\mu \mathrm{U} / \mathrm{ml})$ & $8.60 \pm 9.45$ \\
TC (mg/dl) & $163.61 \pm 39.94$ \\
LDL-C (mg/dl) & $92.75 \pm 28.25$ \\
HDL-C (mg/dl) & $45.72 \pm 10.44$ \\
HA1C (\%) & $7.80 \pm 1.55$ \\
Insulin resistance & $3.28 \pm 4.71$ \\
FBS (mg/dl) & $148.13 \pm 62.57$ \\
\hline & $\mathrm{n} \%$ \\
Gender & \\
Female & $148(63.0)$ \\
\hline Marital status & \\
Single & $9(3.8)$ \\
Married & $215(91.5)$ \\
Widow and divorced & $11(4.7)$ \\
\hline Education & \\
Illiterate & $2(0.9)$ \\
Primary school & $65(27.7)$ \\
Secondary school & $51(21.7)$ \\
High school and diploma & $77(32.8)$ \\
University & $40(17.0)$ \\
\hline Employment & \\
Unemployed and housekeeper & $135(57.4)$ \\
Employed & $52(22.1)$ \\
Retired & $48(20.4)$ \\
\hline Physically active & \\
Yes & $92(39.1)$ \\
\hline BMI, body mass index; BP, blood pressure; FBS, fasting blood sugar; \\
HbAlc, hemoglobin Alc; HDL, high-density lipoprotein cholesterol; LDL- \\
C, low-density lipoprotein cholesterol; TG, triglyceride; $\mathrm{TC}$, total \\
cholesterol. & \\
\hline
\end{tabular}

(DQI-1, HEI-2010, and PI) have been evaluated in none of these studies.

In the present study, there was only a direct correlation between the patients' age and the score of phytochemical index and score of HEI-2010 index; this correlation was statistically significant only with phytochemical index $(p=0.006)$. Also, in other studies, a significant correlation has been reported between age and diet quality indices. For instance, in Mariscal-Arcas's study, there was also a significant correlation between DQI-I and age [20]. Also, in Coltman's study, it was observed that older people had a higher HEI score compared to the patients between 40 and 60 years old. Their results were consistent with ours [21]. Also, in a study carried out by Patricia Guenther, the mean score of HEI-2010 was lower in younger adults compared to older adults; the obtained results were similar to both editions of HEI (HEI-2005 and HEI-2010) [22]. In the studies conducted in Iran, it has also been observed that older participants have higher phytochemical indices [23, 24] although few studies have revealed different results. This is similar to Mabililoni's study, in which reverse and significant 
TABLE 2: Correlation ( $r, p$ value) between age, BMI, and clinical/biochemical variables and three diet quality indices.

\begin{tabular}{|c|c|c|c|}
\hline \multirow[b]{2}{*}{$\begin{array}{l}\text { Variables } r \\
p \text { value }\end{array}$} & \multicolumn{3}{|c|}{ Diet quality indices } \\
\hline & DQI-I & HEI & PI \\
\hline \multirow{2}{*}{ Age } & 0.062 & 0.104 & 0.154 \\
\hline & 0.345 & 0.111 & $0.018 *$ \\
\hline \multirow{2}{*}{ BMI } & -0.22 & -0.123 & -0.131 \\
\hline & 0.740 & 0.059 & $0.045 *$ \\
\hline \multirow{2}{*}{ BP-systolic } & -0.023 & 0.002 & -0.032 \\
\hline & 0.729 & 0.972 & 0.629 \\
\hline \multirow{2}{*}{ BP-diastolic } & -0.062 & 0.11 & -0.095 \\
\hline & 0.348 & 0.869 & 0.146 \\
\hline \multirow{2}{*}{ TG (mg/dl) } & 0.006 & 0.110 & -0.098 \\
\hline & 0.931 & 0.094 & 0.136 \\
\hline \multirow{2}{*}{ Insulin $(\mu \mathrm{U} / \mathrm{ml})$} & -0.034 & -0.050 & -0.098 \\
\hline & 0.602 & 0.449 & 0.135 \\
\hline \multirow{2}{*}{$\mathrm{TC}(\mathrm{mg} / \mathrm{dl})$} & 0.083 & 0.110 & -0.014 \\
\hline & 0.207 & $0.039 *$ & 0.833 \\
\hline \multirow{2}{*}{ LDL-C (mg/dl) } & 0.089 & 0.130 & -0.046 \\
\hline & 0.174 & $0.047 *$ & 0.485 \\
\hline \multirow{2}{*}{ HDL-C (mg/dl) } & 0.046 & 0.058 & 0.129 \\
\hline & 0.485 & 0.378 & $0.048 *$ \\
\hline \multirow{2}{*}{ HbA1c (\%) } & 0.017 & 0.49 & -0.002 \\
\hline & 0.790 & 0.453 & 0.977 \\
\hline \multirow{2}{*}{ Insulin resistance } & 0.064 & 0.113 & 0.093 \\
\hline & 0.801 & 0.884 & 0.102 \\
\hline \multirow{2}{*}{ FBS (mg/dl) } & -0.86 & 0.035 & -0.090 \\
\hline & 0.189 & 0.591 & 0.171 \\
\hline
\end{tabular}

correlation was observed between age and DQI-I score [25]. Therefore, it can be stated that increase in age is correlated with better adherence to nutritional recommendations. One of the possible reasons can be the necessity of the control of chronic diseases present at these ages due to adherence to the diet recommendations which leads to improvement of the quality of diet with increase in age.

There was no significant statistical correlation between the anthropometric indices and blood pressure of type 2 diabetes patients and diet quality assessment indices, in the present study, which were parallel with results of MariscalArcas study [20]; why it is so in this study can be illustrated in that the obesity status and body mass index did not show a significant correlation with DQI-I [25] although the evaluation of DQI-I components was effective in the correlation shown between obesity status and BMI with DQI-I.

However, in the study of Nicklas, it was observed that $\mathrm{BMI}$, waist perimeter, diastolic blood pressure, and score of HEI-2005 index had a reverse correlation. Also, individuals who had the highest diet quality were less affected with obesity or overweight, increase of waist perimeter, and high blood pressure [26]. In the study of Quatromoni, women and men with the highest DQI score had also a lower weight [27]. In fact, the results of this study indicate favorable effects of antioxidants on metabolic disorders, prevention of reweighing, and increase of abdominal obesity [28].
Furthermore, in the study of Mirmiran, the scores higher than phytochemical index had a reverse correlation with 3year alteration of weight, waist perimeter, and body fat [29]. Also, high intake of fruits was correlated with a decrease in overweight. In fact, high score of phytochemical index had favorable effects on prevention of reweighing and decrease of fat in adults. In another study, the participants with higher PI had lower weight and waist perimeter and the risk of abdominal obesity was $66 \%$ lower among them. As a result, it has been observed that high intake of foods rich in phytochemicals had a reverse correlation with abdominal obesity.

4.1. The Correlation between Glycemic Status and Diet Quality Evaluation Indices. In the present study, which was a sectional study for evaluation of the correlation between diet quality and glycemic status in patients with type 2 diabetes, no significant correlations were observed between the scores of the 3 evaluated indices, fasting blood sugar, glycosylated hemoglobin (HbAlc), insulin, and insulin resistance. It is noteworthy that there was a high coherence between the two variables of insulin and insulin resistance $(p<0.001$ and $r=0.901)$; therefore, the independent variable of insulin was replaced with resistance to insulin. Few studies have evaluated the correlation of diet quality and fasting blood sugar 
TABLE 3: DQI-International (DQI-I) components and criteria used for maximum score and zero score $(n=235)$.

\begin{tabular}{|c|c|c|c|}
\hline Components & Score & Scoring criteria & Mean \pm SD \\
\hline DQI-I, total & $0-100$ & - & $58.2 \pm 7.34$ \\
\hline Variety & $0-20$ & - & $19.77 \pm 0.77$ \\
\hline Overall food group variety & $0-15$ & $\begin{array}{l}\geq 1 \text { serving from each food group } / \mathrm{d}=15 \\
\text { Any } 1 \text { food group missing } / \mathrm{d}=12 \\
\text { Any } 2 \text { food groups missing } / \mathrm{d}=9 \\
\text { Any } 3 \text { food groups missing } / \mathrm{d}=6 \\
\geq 4 \text { food groups missing } / \mathrm{d}=3 ; \text { none }=0\end{array}$ & $14.88 \pm 0.64$ \\
\hline $\begin{array}{l}\text { Within-group variety for protein } \\
\text { source }\end{array}$ & $0-5$ & $\begin{array}{l}\geq 3 \text { different sources } / \mathrm{d}=5 ; 2 \text { different sources } / \mathrm{d}=3 \text {; from } 1 \text { source } / \mathrm{d}=1 ; \\
\text { none }=0\end{array}$ & $4.88 \pm 0.46$ \\
\hline Adequacy & $0-40$ & & $25.98 \pm 5.53$ \\
\hline Vegetable group & $0-5$ & $\geq 3-5$ servings $/ d=5 ; 0$ servings $/ d=0$ & $1.42 \pm 2.26$ \\
\hline Fruit group & $0-5$ & $\geq 2-4$ servings $/ d=5 ; 0$ servings $/ d=0$ & $1.00 \pm 2.01$ \\
\hline Grain group & $0-5$ & $\geq 6-11$ servings $/ d=5 ; 0$ servings $/ d=0$ & $4.03 \pm 1.98$ \\
\hline Fiber & $0-5$ & $\geq 20-30 \mathrm{~g} / \mathrm{d}=5 ; 0 \mathrm{~g} / \mathrm{d}=0$ & $3.53 \pm 2.28$ \\
\hline Protein & $0-5$ & $\geq 10 \%$ of energy/d $=5 ; 0 \%$ of energy $/ d=0$ & $4.90 \pm 0.10$ \\
\hline Iron & $0-5$ & $\geq 100 \%$ of $\mathrm{RDA}(\mathrm{AI}) / \mathrm{d}=5 ; 0 \%$ of $\mathrm{RDA}(\mathrm{AI}) / \mathrm{d}=0$ & $4.98 \pm 0.26$ \\
\hline Calcium & $0-5$ & $\geq 10 \%$ of $\mathrm{AI} / \mathrm{d}=5 ; 0 \%$ of $\mathrm{AI} / \mathrm{d}=0$ & $4.97 \pm 0.32$ \\
\hline Vitamin C & $0-5$ & $\geq 100 \%$ of $\mathrm{RDA}(\mathrm{RNI}) / \mathrm{d}=5 ; 0 \%$ of $\mathrm{RDA}(\mathrm{RNI}) / \mathrm{d}=0$ & $4.70 \pm 0.80$ \\
\hline Moderation & 0-30 & & $12.45 \pm 4.35$ \\
\hline Total fat & $0-6$ & $\leq 20 \%$ of total energy $(\mathrm{TE}) / \mathrm{d}=6 ;>20-30 \%$ of $\mathrm{TE} / \mathrm{d}=3 ;>30 \%$ of $\mathrm{TE} / \mathrm{d}=0$ & $3.13 \pm 2.09$ \\
\hline Saturated fat & $0-6$ & $\leq 7 \%$ of $\mathrm{TE} / \mathrm{d}=6 ;>7-10 \%$ of $\mathrm{TE} / \mathrm{d}=3 ;>10 \%$ of $\mathrm{TE} / \mathrm{d}=0$ & $1.06 \pm 0.71$ \\
\hline Cholesterol & $0-6$ & $\leq 300 \mathrm{mg} / \mathrm{d}=6 ;>300-400 \mathrm{mg} / \mathrm{d}=3 ;>400 \mathrm{mg} / \mathrm{d}=0$ & $5.41 \pm 1.48$ \\
\hline Sodium & $0-6$ & $\leq 2,400 \mathrm{mg} / \mathrm{d}=6 ;>2,400-3,400 \mathrm{mg} / \mathrm{d}=3 ;>3,400 \mathrm{mg} / \mathrm{d}=0$ & $0.87 \pm 1.80$ \\
\hline Empty calorie foods & $0-6$ & $3 \%$ of $\mathrm{TE} / \mathrm{d}=6 ;>3-10 \%$ of $\mathrm{TE} / \mathrm{d}=3 ;>10 \%$ of $\mathrm{TE} / \mathrm{d}=0$ & $3.04 \pm 2.47$ \\
\hline Overall balance & 0-10 & & $0.61 \pm 1.71$ \\
\hline Macronutrient ratio & $0-6$ & $\begin{array}{c}55-65: 10-15: 15-25=6 ; 52-68: 9-16: 13-27=4 ; 50-70: 8-17: 12-30=2 ; \\
\text { otherwise }=0\end{array}$ & $0.25 \pm 1.21$ \\
\hline Fatty acid ratio & $0-4$ & $\begin{aligned} \mathrm{P} / \mathrm{S}=1-1.5 \text { and } \mathrm{M} / \mathrm{S}=1-1.5= & 4 ; \text { else if } \mathrm{P} / \mathrm{S}=0.8-1.7 \text { and } \mathrm{M} / \mathrm{S}=0.8-1.7=2 ; \\
& \text { otherwise }=0\end{aligned}$ & $0.36 \pm 1.11$ \\
\hline
\end{tabular}

AI, adequate intake; d, day; M, monounsaturated fatty acid; P, polyunsaturated fatty acid, RDA, Recommended Dietary Allowances; S, saturated fatty acid; TE, total energy.

TABle 4: Healthy Eating Index (HEI-2010) components and criteria used for maximum score and zero score $(n=235)$.

\begin{tabular}{|c|c|c|c|}
\hline Components & Score & Scoring criteria & Mean \pm SD \\
\hline HEI, total score & $0-100$ & & $55.83 \pm 10.61$ \\
\hline Adequacy & $0-60$ & & $12.71 \pm 1.12$ \\
\hline Total fruit & $0-5$ & $\geq 0.8$ cup equiv. per $1,000 \mathrm{kcal}$; no fruit $=0$ & $1.23 \pm 0.94$ \\
\hline Whole fruit & $0-5$ & $\geq 0.4$ cup equiv. per $1,000 \mathrm{kcal}$; no whole fruit $=0$ & $0.92 \pm 0.70$ \\
\hline Total vegetables & $0-5$ & $\geq 1.1$ cup equiv. per $1,000 \mathrm{kcal}$; no vegetables $=0$ & $1.85 \pm 1.16$ \\
\hline Greens and beans & $0-5$ & $\geq 0.2$ cup equiv. per $1,000 \mathrm{kcal}$; no dark green vegetables or beans and peas $=0$ & $0.24 \pm 0.26$ \\
\hline Whole grains & $0-10$ & $\geq 1.5 \mathrm{oz}$ equiv. per $1,000 \mathrm{kcal}$; no whole grains $=0$ & $1.55 \pm 1.90$ \\
\hline Dairy & $0-10$ & $\geq 1.3$ cup equiv. per $1,000 \mathrm{kcal}$; no dairy $=0$ & $0.35 \pm 0.31$ \\
\hline Total protein foods & $0-5$ & $\geq 2.5 \mathrm{oz}$ equiv. per $1,000 \mathrm{kcal}$; no protein foods $=0$ & $2.96 \pm 2.24$ \\
\hline Seafood and plant proteins & $0-5$ & $\geq 0.8 \mathrm{oz}$ equiv. per $1,000 \mathrm{kcal}$; no seafood or plant proteins $=0$ & $1.18 \pm 1.59$ \\
\hline Fatty acids & $0-10$ & (PUFAs + MUFAs)/SFAs $\geq 2.5 ;$ (PUFAs + MUFAs)/SFAs $\leq 1.2$ & $2.42 \pm 0.80$ \\
\hline Moderation & $0-40$ & & $6.15 \pm 1.21$ \\
\hline Refined grains & 10 & $\leq 1.8 \mathrm{oz}$ equiv. per $1,000 \mathrm{kcal} ; \geq 4.3 \mathrm{oz}$ equiv. per $1,000 \mathrm{kcal}$ & $5.04 \pm 3.47$ \\
\hline Sodium & 10 & $\leq 1.1$ grams per $1,000 \mathrm{kcal} ; \geq 2.0$ grams per $1,000 \mathrm{kcal}$ & $0.90 \pm 1.01$ \\
\hline Empty calories & 20 & $\leq 19 \%$ of energy; $\geq 50 \%$ of energy & $0.11 \pm 0.17$ \\
\hline
\end{tabular}

HEI, Healthy Eating Index; MUFA, monounsaturated fatty acid; PUFA, polyunsaturated fatty acid; SFA, saturated fatty acid.

and insulin, and most of them have evaluated the correlation of the risk of diabetes and scores of diet quality indices. In the study conducted by Juturu (2006), the serum insulin levels and insulin resistance had a significant correlation with HEI scores in the patients with type 2 diabetes [30]. In another study by Chiuve (2012), there was a low correlation between AHEI-2010 and HEI-2005 with coronary artery diseases and type 2 diabetes. The results of this study showed that adherence to the HEI-2005 recommendations might decrease the risk of chronic diseases, but AHEI-2010, which 
includes more nutritional information, had a higher correlation with the risk of chronic diseases, especially CHD and diabetes [31]. In the study of Ferranti, higher AHEI score had also a reverse correlation with $\mathrm{HbA1C}$, indicating the protective effect of adherence to AHEI recommendations on cardiometabolic factors in women with a history of GDM (pregnancy diabetes) [32]. Also, in another study, similar results were observed and the incidence of fasting blood sugar disorder was decreased by $75 \%$ in men with higher HEI scores [7]. Further, consumption of mixed diets of fruits and vegetables had a high reverse correlation with the incidence of type 2 diabetes; these findings indicate the importance of consumption of fruits and vegetables in prevention of type 2 diabetes [33].

The results of the present study indicated a direct correlation between serum LDL-C levels and the score of HEI2010 index and DQI-I index which had only significant correlation with HEI-2010 index $(p=0.026)$. In the study carried out by Nicklas, HEI-2005 score had a reverse correlation with total cholesterol and LDL and a direct and significant correlation with HDL-C. Furthermore, people with the highest diet quality were less affected with decrease in HDL [26]. In another study, HEI-2005 index was applied for evaluation of the quality of diet, and it was observed that people with higher percentile of HEI had lower LDL and triglyceride and higher HDL [34].

Studies performed in Iran also reported similar results. In a study conducted by Asghari et al., it was reported that men with the highest score of HEI-2005 index had significantly lower levels of serum triglyceride although this correlation was not seen among women. On the other hand, a direct correlation was observed in men between DQI-1 and HDL-C scores. Moreover, in both genders, all three indices had a weak correlation with total cholesterol, while no correlation was observed between the indices with LDL-C. In fact, HEI-2005 index had a weak correlation with a decrease in serum levels of triglyceride in urban population [10]. According to animal and human studies, it has been reported that herbal polyphenols in diet and consumption of products rich in polyphenols lead to adjustment of carbohydrate and fat metabolism, decrease in the level of sugar, decrease in disorders of blood fat, reduction of insulin resistance, improvement of adipose tissue metabolism, and decrease in the oxidative stress and inflammation. Polyphenolic contents can prevent development of chronic complications of diabetes including cardiovascular diseases, nephropathy, neuropathy, and retinopathy [35].

While most of the previous studies had reported a reverse and significant correlation between diet quality and serum levels of LDL-C, the results of the present study indicated a direct and significant correlation between them; it seems that the reason for the contradiction about the correlation between the score of diet quality index and lipid profile with previous studies is that, in the present study, most patients consumed blood fat decreasing drugs such as atorvastatin that this might have been a confounding factor as to this correlation. On the other hand, in the present study, the duration of having diabetes in participants was not considered, while this can be one of the other confounding factors. Also, over time and due to better control of disease and its complications, the participants directed their diets into having a more healthy diet. Also, the nature of type 2 diabetes is the other reason for justifying the increase in the serum levels of LDL-C in patients participating in the present study. It is noteworthy that, in previous studies, most of the communities evaluated had been healthy people, while in the present study the subjects were patients with type 2 diabetes.

In the present study, PI, HEI-2010 and DQI-I indices had a weak correlation with each other and only the correlation between PI and DQI-I indices was significant $(p<0.001$ and $r=0.232$ ).

Very limited studies have evaluated diet quality indices simultaneously. Therefore, the available results are few in order to evaluate and compare them with the results of the present study. In one of these limited studies, although a direct correlation was seen in men between scores of DQI-I and HDL-C, in both genders, all the three indices (HEI-2005, MDS, and DQI-I) had a weak correlation with total cholesterol. However, no correlation was observed between the indices with serum LDL-C. Only HEI-2005 index had a weak correlation with the decline in serum triglyceride concentrations among the urban population of Iran, but there was no correlation between the two indices of MDS and DQI-I with lipid profile. Although, in this study, the correlation between indices was not evaluated directly, the results of the study indicated a weak correlation between these indices. Therefore, it seems that the intended indices for diet quality evaluation have a weak and insignificant correlation with each other. Furthermore, since the present study is the only study reporting the significant correlation of PI and DQI indices, it is essential to conduct more studies to confirm this aspect.

Our study had some limitations including low sample size, no long-term follow up of the patients, and no consideration of the duration of diabetes disease and drug regimens in patients as a confounding factor.

\section{Conclusions}

Generally, it seems that inconsistency of the findings in the present study with some previous studies is due to the difference in nutritional habits and type and amount of meals among various countries. Why it is so even in similar amounts is that the quality and nutritional values are not similar and also the diet quality evaluation indices are formed based on the information of societies which have numerous differences with our population of the study.

\section{Data Availability}

Data are available upon request to the corresponding author.

\section{Conflicts of Interest}

The authors declare no conflicts of interest. 


\section{Authors' Contributions}

All authors had equal roles in design, work, statistical analysis, and manuscript writing.

\section{Acknowledgments}

This study was funded by the Vice-Chancellor of Research at the Shiraz University of Medical Sciences, Shiraz, Iran.

\section{References}

[1] I. Hameed, S. R. Masoodi, S. A. Mir, M. Nabi, K. Ghazanfar, and B. A. Ganai, "Type 2 diabetes mellitus: from a metabolic disorder to an inflammatory condition," World Journal of Diabetes, vol. 6, no. 4, p. 598, 2015.

[2] S. A. Tabish, "Is diabetes becoming the biggest epidemic of the twenty-first century?" International Journal of Health Sciences, vol. 1, no. 2, 2007.

[3] H. P. Fransen and M. C. Ocké, "Indices of diet quality," Current Opinion in Clinical Nutrition and Metabolic Care, vol. 11, no. 5, pp. 559-565, 2008.

[4] G. M. Turner-McGrievy, N. D. Barnard, J. Cohen, D. J. A. Jenkins, L. Gloede, and A. A. Green, "Changes in nutrient intake and dietary quality among participants with type 2 diabetes following a low-fat vegan diet or a conventional diabetes diet for 22 weeks," Journal of the American Dietetic Association, vol. 108, no. 10, pp. 1636-1645, 2008.

[5] S. A. McNaughton, D. W. Dunstan, K. Ball, J. Shaw, and D. Crawford, "Dietary quality is associated with diabetes and cardio-metabolic risk factors," The Journal of Nutrition, vol. 139, no. 4, pp. 734-742, 2009.

[6] T. R. Nansel, D. L. Haynie, L. M. Lipsky, L. M. B. Laffel, and S. N. Mehta, "Multiple indicators of poor diet quality in children and adolescents with type 1 diabetes are associated with higher body mass index percentile but not glycemic control," Journal of the Academy of Nutrition and Dietetics, vol. 112, no. 11, pp. 1728-1735, 2012.

[7] B. Gopinath, E. Rochtchina, V. M. Flood, and P. Mitchell, "Diet quality is prospectively associated with incident impaired fasting glucose in older adults," Diabetic Medicine, vol. 30, no. 5, pp. 557-562, 2013.

[8] S. L. Teoh and S. Das, "Phytochemicals and their effective role in the treatment of diabetes mellitus: a short review," Phytochemistry Reviews, vol. 17, no. 5, pp. 1111-1128, 2018.

[9] R. Rahimi, S. Nikfar, B. Larijani, and M. Abdollahi, "A review on the role of antioxidants in the management of diabetes and its complications," Biomedicine \& Pharmacotherapy, vol. 59, no. 7, pp. 365-373, 2005.

[10] G. Asghari, P. Mirmiran, F. Hosseni-Esfahani, P. Nazeri, M. Mehran, and F. Azizi, "Dietary quality among tehranian adults in relation to lipid profile: findings from the tehran lipid and glucose study," Journal of Health, Population, and Nutrition, vol. 31, no. 1, p. 37, 2013.

[11] F. G. Huffman, M. De La Cera, J. A. Vaccaro et al., "Healthy eating index and alternate healthy eating index among Haitian Americans and African Americans with and without type 2 diabetes," Journal of Nutrition and Metabolism, vol. 2011, Article ID 398324, 8 pages, 2011.

[12] K. L. Piercy, R. P. Troiano, R. M. Ballard et al., "The physical activity guidelines for Americans," The Journal of the American Medical Association, vol. 320, no. 19, pp. 2020$2028,2018$.
[13] National Cancer Institute Dap, 24-hour Dietary Recall (24HR) at a Glance, National Cancer Institute Dap, Bethesda, MD, USA, 2020, https://dietassessmentprimer.cancer.gov/profiles/ recall/.

[14] A. V. Bennett, D. L. Patrick, D. M. Bushnell, C. F. Chiou, and P. Diehr, "Comparison of 7-day and repeated 24-h recall of type 2 diabetes," Quality of Life Research, vol. 20, no. 5, pp. 769-777, 2011.

[15] F. Roshanzamir, M. Miraghajani, M. Mansourian, R. Ghiasvand, and S. M. Safavi, "Association between healthy eating index-2010 and fetuin-A levels in patients with type 2 diabetes: a case-control study," Clinical Nutrition Research, vol. 6, no. 4, pp. 296-305, 2017.

[16] S. Kim, P. S. Haines, A. M. Siega-Riz, and B. M. Popkin, "The Diet Quality Index-International (DQI-I) provides an effective tool for cross-national comparison of diet quality as illustrated by China and the United States," The Journal of Nutrition, vol. 133, no. 11, pp. 3476-3484, 2003.

[17] M. F. McCarty, "Proposal for a dietary "phytochemical index"” Medical Hypotheses, vol. 63, no. 5, pp. 813-817, 2004.

[18] F. G. Huffman, J. A. Vaccaro, J. C. Exebio, G. G. Zarini, T. Katz, and Z. Dixon, “Television watching, diet quality, and physical activity and diabetes among three ethnicities in the United States," Journal of Environmental and Public Health, vol. 2012, Article ID 191465, 10 pages, 2012.

[19] C. S. Fox, S. H. Golden, C. Anderson et al., "Update on prevention of cardiovascular disease in adults with type 2 diabetes mellitus in light of recent evidence," Circulation, vol. 132, no. 8, pp. 691-718, 2015.

[20] M. Mariscal-Arcas, D. Romaguera, A. Rivas et al., "Diet quality of young people in southern Spain evaluated by a Mediterranean adaptation of the Diet Quality Index-International (DQI-I)," British Journal of Nutrition, vol. 98, no. 6, pp. 1267-1273, 2007.

[21] A. E. Coltman, K. S. Keim, K. M. Chapman-Novakofski, and C. A. Taylor, "Assessing diet quality of a type 2 diabetes sample using the Healthy Eating Index 2005," Topics in Clinical Nutrition, vol. 28, no. 2, pp. 145-153, 2013.

[22] P. M. Guenther, S. I. Kirkpatrick, J. Reedy et al., "The healthy eating index-2010 is a valid and reliable measure of diet quality according to the 2010 dietary guidelines for Americans," The Journal of Nutrition, vol. 144, no. 3, pp. 399-407, 2013.

[23] Z. Bahadoran, M. Golzarand, P. Mirmiran, N. Saadati, and F. Azizi, "The association of dietary phytochemical index and cardiometabolic risk factors in adults: tehran lipid and glucose study," Journal of Human Nutrition and Dietetics, vol. 26, no. s1, pp. 145-153, 2013.

[24] M. Golzarand, P. Mirmiran, Z. Bahadoran, S. Alamdari, and F. Azizi, "Dietary phytochemical index and subsequent changes of lipid profile: a 3-year follow-up in tehran lipid and glucose study in Iran," ARYA Atherosclerosis, vol. 10, no. 4, p. 203, 2014.

[25] M. del Mar Bibiloni, A. Pons, and J. A. Tur, "Diet quality of Mediterranean adolescents evaluated by Mediterranean adaptation of the Diet Quality Index-Internationa (DQI I): socioeconomic, anthropometric, lifestyle and body image determinants," Journal of Clinical Nutrition \& Dietetics, vol. 1, no. $1,2015$.

[26] T. A. Nicklas, C. E. O’Neil, and V. L. Fulgoni III, "Diet quality is inversely related to cardiovascular risk factors in adults," The Journal of Nutrition, vol. 142, no. 12, pp. 2112-2118, 2012.

[27] P. A. Quatromoni, M. Pencina, M. R. Cobain, P. F. Jacques, and R. B. D'Agostino, "Dietary quality predicts adult weight 
gain: findings from the framingham offspring study," Obesity, vol. 14, no. 8, pp. 1383-1391, 2006.

[28] Z. Bahadoran, M. Golzarand, P. Mirmiran, N. Shiva, and F. Azizi, "Dietary total antioxidant capacity and the occurrence of metabolic syndrome and its components after a 3year follow-up in adults: tehran Lipid and Glucose Study," Nutrition \& Metabolism, vol. 9, no. 1, p. 70, 2012.

[29] P. Mirmiran, Z. Bahadoran, M. Golzarand, N. Shiva, and F. Azizi, "Association between dietary phytochemical index and 3-year changes in weight, waist circumference and body adiposity index in adults: tehran lipid and glucose study," Nutrition \& Metabolism, vol. 9, no. 1, p. 108, 2012.

[30] V. Juturu, M. Finch, J. Geohas, and J. R. Komorowski, "Assessment of diet quality in type 2 diabetes using the healthy eating index," The FASEB Journal, vol. 20, no. 5, p. A1313, 2006.

[31] S. E. Chiuve, T. T. Fung, E. B. Rimm et al., "Alternative dietary indices both strongly predict risk of chronic disease," The Journal of Nutrition, vol. 142, no. 6, pp. 1009-1018, 2012.

[32] E. P. Ferranti, S. B. Dunbar, C. M. Reilly, J. W. Foster, and Y. Guo, Diet Quality and Cardiometabolic Risk Status of Women within Five Years Following Gestational Diabetes, American Heart Association, Dallas, TX, USA, 2013.

[33] A. J. M. Cooper, S. J. Sharp, R. N. Luben, K.-T. Khaw, N. J. Wareham, and N. G. Forouhi, "The association between a biomarker score for fruit and vegetable intake and incident type 2 diabetes: the EPIC-Norfolk study," European Journal of Clinical Nutrition, vol. 69, no. 4, p. 449, 2015.

[34] B. S. Shah, J. Cahill, H. Lu, and J. H. Freeland-Graves, "The Healthy Eating Index scores of low-income women in early postpartum and its association with lipid profiles," The FASEB Journal, vol. 23, no. 1 Supplement, pp. 214-222, 2009.

[35] Z. Bahadoran, P. Mirmiran, and F. Azizi, "Dietary polyphenols as potential nutraceuticals in management of diabetes: a review," Journal of Diabetes \& Metabolic Disorders, vol. 12, no. 1, p. 43, 2013. 